\title{
Activation of the Calcium-Sensing Receptor Corrects the Impaired Mitochondrial Energy Status Observed in Renal Polycystin-1 Knockdown Cells Modeling Autosomal Dominant Polycystic Kidney Disease
}

\section{Annarita Di Mise ${ }^{1}$, Marianna Ranieri ${ }^{1}$, Mariangela Centrone ${ }^{1}$, Maria Venneri ${ }^{1}$, Grazia Tamma $^{1,2}$, Daniela Valenti ${ }^{3 *}$ and Giovanna Valenti ${ }^{1,2,4 *}$}

OPEN ACCESS

Edited by:

Graça Soveral,

Universidade de Lisboa, Portugal

Reviewed by:

Kamel Laghmani,

INSERM U1138 Centre de Recherche

des Cordeliers, France

Francesco Moccia,

University of Pavia, Italy

*Correspondence:

Giovanna Valenti

giovanna.valenti@uniba.it

Daniela Valenti

d.valenti@ibiom.cnr.it

Specialty section

This article was submitted to

Cellular Biochemistry,

a section of the journal

Frontiers in Molecular Biosciences

Received: 23 April 2018 Accepted: 03 August 2018

Published: 24 August 2018

Citation:

Di Mise A, Ranieri M, Centrone M, Venneri $M$, Tamma G, Valenti $D$ and

Valenti $G$ (2018) Activation of the Calcium-Sensing Receptor Corrects

the Impaired Mitochondrial Energy Status Observed in Renal Polycystin-1 Knockdown Cells Modeling Autosomal Dominant Polycystic Kidney Disease.

Front. Mol. Biosci. 5:77.

doi: 10.3389/fmolb.2018.00077
${ }^{1}$ Department of Biosciences, Biotechnologies and Biopharmaceutics, University of Bari, Bari, Italy, ${ }^{2}$ Istituto Nazionale di Biostrutture e Biosistemi, Rome, Italy, ${ }^{3}$ Institute of Biomembranes Bioenergetics and Molecular Biotechnologies, National Council of Research, Bari, Italy, ${ }^{4}$ Center of Excellence in Comparative Genomics, University of Bari, Bari, Italy

Autosomal Dominant Polycistic kidney Disease (ADPKD) is a renal channelopathy due to loss-of-function mutations in the PKD1 or PKD2 genes, encoding polycystin-1 (PC1) or polycystin-2 (PC2), respectively. PC1 is a large protein found predominantly on the plasma membrane where interacts with different proteins, including PC2. PC2 is a smaller integral membrane protein also expressed in intracellular organelles, acting as a non-selective cation channel permeable to calcium. Both PC1 and PC2 are also localized to the primary cilium of renal epithelial cells serving as mechanosensor that controls calcium influx through the plasma membrane and regulates intracellular calcium release from the endoplasmic reticulum. The mechanisms by which PC1/2 dysfunction leads to ADPKD needs still to be clarified. We have recently reported that selective Calcium-Sensing Receptor (CaSR) activation in human conditionally immortalized Proximal Tubular Epithelial cells deficient for PC1 (ciPTEC-PC1KD), deriving from urine sediments reduces intracellular CAMP and mTOR activity, and increases intracellular calcium reversing the principal ADPKD dysregulations. Reduced cellular free calcium found in ADPKD can, on the other hand, affect mitochondrial function and ATP production and, interestingly, a relationship between mitochondria and renal polycystic diseases have been suggested. By using ciPTEC-PC1KD as experimental tool modeling of ADPKD, we show here that, compared with wild type cells, ciPTEC-PC1KD have significantly lower mitochondrial calcium levels associated with a severe deficit in mitochondrial ATP production, secondary to a multilevel impairment of oxidative phosphorylation. Notably, selective CaSR activation with the calcimimetic NPS-R568 increases mitochondrial calcium content close to the levels found in resting wild type cells, and fully recovers the cell energy deficit associated to the PC1 channel disruption. Treatment of ciPTEC-PC1KD with 2-APB, an IP3R inhibitor, prevented the 
rescue of bioenergetics deficit induced by CaSR activation supporting a critical role of IP3Rs in driving ER-to-mitochondria Ca2+ shuttle. Together these data indicate that, besides reversing the principal dysregulations considered the most proximal events in ADPKD pathogenesis, selective CaSR activation in PKD1 deficient cells restores altered mitochondrial function that, in ADPKD, is known to facilitate cyst formation. These findings identify CaSR as a potential therapeutic target.

Keywords: calcium-sensing receptor, renal channelopathies, calcimimetics, ciPTEC, mitochondria, ATP

\section{INTRODUCTION}

Loss-of-function of polycystin-1 (PC1) or polycystin-2 (PC2), two trans-membrane proteins that form a heteromeric molecular complex in the cilia and plasma membrane, results in the most common life-threatening genetic renal disorder, called Autosomal Dominant Polycystic Kidney Disease (ADPKD), characterized by the formation and development of kidney cysts (Torres et al., 2007). Biochemical experiments have shown that PC1 and PC2 form a receptor-ion channel complex (Hanaoka et al., 2000; Yu et al., 2009) leading ADPKD to be classified among channelopathies, a heterogeneous group of disorders caused by the dysfunction of ion channels expressed in cellular membranes and many intracellular organelles.

ADPKD affects 1:400-1:1,000 worldwide and 50\% of PKD patients will require dialysis or kidney transplantation within 60 years of age (Harris and Torres, 2014). It results from mutations in PKD1 or PKD2 genes (encoding PC1 or PC2, respectively) (Hughes et al., 1995; Mochizuki et al., 1996) which cause analogous clinical signs. The close identity of the disease manifestations, independently of the responsible gene, suggests that $\mathrm{PC} 1$ and $\mathrm{PC} 2$ are involved in a common signaling pathway.

PC1 is considered a mechanosensor receptor with a large extracellular $\mathrm{N}$-terminal, 11 transmembrane domains and a cytoplasmic C-terminal region (Hughes et al., 1995; Oatley et al., 2012). The $\mathrm{N}$-terminal domain contains several structural motifs found to be essential for interactions with extracellular proteins and carbohydrates (Löhning et al., 1996). The Cterminal cytoplasmic domain contains a coiled-coil sequence that binds specifically to the C-terminus of PC2 (Qian et al., 1997), and it has been shown that, once cleaved from the full length PC1, it translocates to the nucleus (Chauvet et al., 2004; Chapin et al., 2010). Interestingly, PC2 expression is able to attenuate PC1 C-terminal tail translocation, modulating its signaling properties (Chauvet et al., 2004).

PC2, also known as transient receptor potential cation channel, subfamily P, member 2 (TRPP2), is a nonselective cation channel containing 6 transmembrane domains, with both $\mathrm{N}$ and C-terminal tails facing the cytoplasm (Mochizuki et al., 1996; Celić et al., 2008). The N-terminal portion carries a ciliary targeting domain (Geng et al., 2006), while the C-terminal tail includes an EF-hand, and a coiled coil motif whose binding to the coiled-coil domain in the PC1 C terminus is critical for the formation of the PC1-PC2 complex (Yu et al., 2009). Conversely, other experiments showed that PC1 and PC2 interaction is preserved in systems not expressing the coiled-coil domain and is dependent on the N-terminal domain (Babich et al., 2004; Feng et al., 2008; Celić et al., 2012). However, the factors that regulate the localization, trafficking, and channel activity of the polycystins remain still unclear.

The polycystins form a complex that localizes to the primary cilium, where it may be involved in chemosensory or mechanosensory pathways (Pazour and Rosenbaum, 2002; Chapin and Caplan, 2010). In addition, the PC complex is expressed on the plasma membrane, as well as in intracellular compartments, where $\mathrm{PC} 2$ can regulate $\mathrm{Ca}^{2+}$ release from the endoplasmic reticulum (Koulen et al., 2002). Many studies propose that PC1 and PC2 may reciprocally affect each other's membrane or ciliary localization (Harris et al., 1995; Ong and Wheatley, 2003; Xu et al., 2007), and importantly, the interaction between the polycystins has been suggested to be crucial in defining the properties of the ion channel associated with the complex (Hanaoka et al., 2000; Delmas et al., 2004).

In ADPKD, disruption of the PC complex, resulting from the loss of function of $P K D 1$ or $P K D 2$, cause the dysregulation of many pathways such as cAMP, calcium and mTOR signaling cascades, which promote cell proliferation and apoptosis. The hallmark of the disease is the spontaneous generation and constant growth of kidney cysts which gradually expand to demolish the normal renal parenchyma, leading to end stage renal disease (ESRD).

We have recently shown that selective activation of the extracellular calcium-sensing receptor (CaSR) in human conditionally immortalized proximal tubular epithelial cells (ciPTEC), silenced for PKD1 or generated from an ADPKD1 patient, increases cytosolic calcium, and reduces intracellular cAMP and mTOR activity, reversing the principal dysregulations considered the most proximal events in ADPKD pathogenesis, making CaSR a possible candidate as therapeutic target (Di Mise et al., 2018). On the other hand, reduced intracellular calcium observed in ADPKD can affect mitochondrial function and ATP production with consequence on glucose metabolism (Rowe et al., 2013), as showed in recent studies which reported defective glucose metabolism in ADPKD-affected cells and tissues (Rowe et al., 2013; Menezes et al., 2016). They demonstrated that PC1 lacking cells consume high levels of glucose, preferentially using it in aerobic glycolysis for their energy production (Rowe et al., 2013). Indeed, mitochondrial abnormality exists from an early phase of ADPKD, underlining a relationship between mitochondria and renal polycystic diseases ( $\mathrm{Li}$ et al., 2012; Rowe et al., 2013; Magistroni and Boletta, 2017; Padovano et al., 2017). In line, an association between mitochondria 
abnormalities and cystogenesis has been reported in cyst-lining cells in ADPKD model mice and in rats (Ishimoto et al., 2017). Recent clinical studies indicate that oxidative stress is already present in the early stages of ADPKD, even when renal function is preserved (Menon et al., 2011; Klawitter et al., 2014), showing that mitochondria, representing the primary reactive oxygen species source, play a functional role in cyst formation. However, the precise pathophysiological role of mitochondria in ADPKD remains elusive.

We now report that CaSR activation in ciPTEC stably knocked down for PC1 restores the decreased mitochondrial calcium levels and fully reverse the deficient ATP production.

\section{MATERIALS AND METHODS}

\section{Materials}

All chemicals were purchased from Sigma (Sigma-Aldrich, Milan, Italy). NPS-R568 was kindly gifted by Amgen (Amgen Dompé S.p.a., Milan, Italy). Media for cell culture were from Lonza (Lonza s.r.l., Milan, Italy). pcDNA-4mtD3cpv was a gift from Amy Palmer \& Roger Tsien (Addgene plasmid \#36324).

\section{Antibodies}

Monoclonal CaSR antibody recognizing amino-acid 15-29 at the extracellular N-terminus was from Sigma-Aldrich, Milan, Italy. Secondary goat anti-mouse IgG biotin antibodies were purchased from Sigma-Aldrich, Milan, Italy. Streptavidin-488 conjugate were from Alexa Fluor (Molecular Probes, Eugene, Oregon, USA).

\section{Generation of ciPTEC Knocked Down for Polycystin-1}

ciPTEC were generated as described by Wilmer et al. (2010). Briefly, primary cells were cultured by collecting mid-stream urine within $5 \mathrm{~h}$ after collection. Urine sediment was resuspended in DMEM Ham's F12 medium supplemented with $10 \%$ fetal bovine serum (FBS), $100 \mathrm{IU} / \mathrm{ml}$ penicillin, $100 \mu \mathrm{g} / \mathrm{ml}$ streptomycin, ITS $(5 \mu \mathrm{g} / \mathrm{ml}$ insulin, $5 \mu \mathrm{g} / \mathrm{ml}$ transferrin and $5 \mathrm{ng} / \mathrm{ml}$ selenium), $36 \mathrm{ng} / \mathrm{ml}$ hydrocortisone, $10 \mathrm{ng} / \mathrm{ml}$ epidermal growth factor (EGF), and $40 \mathrm{pg} / \mathrm{ml}$ triiodothyronine. The suspension was placed at $37^{\circ} \mathrm{C}$ in a $5 \% \mathrm{CO}_{2}$ incubator.

Primary cells were immortalized as previously described (Wilmer et al., 2010). Cells were infected with SV40T and hTERT vectors, containing, respectively, geneticin (G418) and hygromycin resistance (O'hare et al., 2001; Satchell et al., 2006). Subconfluent cell layers were then grown at $33^{\circ} \mathrm{C}$ and selected by using G418 $(400 \mu \mathrm{g} / \mathrm{ml})$ and hygromycin B $(25 \mu \mathrm{g} / \mathrm{ml})$ for 10 days. Stable knocked down ciPTEC for polycystin-1 (ciPTECPC1KD) were obtained transducing a cloned ciPTEC line (ciPTECwt) of a healthy individual (34.8) by adding lentiviral vectors encoding miR-shRNA directed against polycystin-1, cloned in tandem (pCHMWS Bsd 2xmiRNA PKD1), to the culture medium (Mekahli et al., 2012). Transduced cells were selected using $10 \mathrm{~g} / \mathrm{ml}$ blasticidin. Experiments were performed prior cellular maturation for 11 days at $37^{\circ} \mathrm{C}$. The reduced expression of PC1 was showed by Mekahli and coworkers which biochemically characterized these cell lines (Mekahli et al., 2012).

\section{Measurement of Mitochondrial ATP Production Rate in ciPTEC}

The rate of mitochondrial ATP production was determined in ciPTEC permeabilized with digitonin (0.01\%) essentially as already reported (Valenti et al., 2016). Briefly, cells (0.3$0.4 \mathrm{mg}$ protein) were incubated at $37^{\circ} \mathrm{C}$ in $2 \mathrm{ml}$ of respiratory medium consisting of $210 \mathrm{mM}$ mannitol, $70 \mathrm{mM}$ sucrose, $3 \mathrm{mM}$ $\mathrm{MgCl}_{2}, 20 \mathrm{mM}$ Tris/ $\mathrm{HCl}, 5 \mathrm{mM} \mathrm{KH}{ }_{2} \mathrm{PO}_{4} / \mathrm{K}_{2} \mathrm{HPO}_{4},(\mathrm{pH} 7.4)$ plus $5 \mathrm{mg} / \mathrm{ml} \mathrm{BSA}$, in the presence of the coupled enzyme system revealing ATP, indicated as ATP detecting system (ATP$\mathrm{ds})$, containing the substrates glucose $(2.5 \mathrm{mM})$ and $\mathrm{NADP}^{+}$ $(0.25 \mathrm{mM})$ and the coupled enzymes hexokinase (HK, 3 e.u.) and glucose 6-phosphate dehydrogenase (G6P-DH, 2 e.u.). The assay has been performed in the presence of $0.01 \mathrm{mM}$ diadenosine pentaphosphate (Ap5A) to selectively inhibit the mitochondrial adenylate kinase. The measure has been carried out by adding as energy sources, either glutamate (GLU) plus malate (MAL) ( $5 \mathrm{mM}$ each) or succinate (SUCC, $5 \mathrm{mM}$ ) plus rotenone (ROT, $3 \mu \mathrm{M}$ ), or ascorbate (ASC, $0.5 \mathrm{mM}$ ) plus $\mathrm{N}, \mathrm{N}, \mathrm{N}^{\prime}, \mathrm{N}^{\prime}$ - tetramethyl-p-phenylenediamine (TMPD, $0.25 \mathrm{mM}$ ). After $5 \mathrm{~min}$ of incubation with digitonin $(0.01 \% \mathrm{w} / \mathrm{v})$, ADP $(0.5 \mathrm{mM})$ was added to start the reaction, and the reduction of $\mathrm{NADP}^{+}$in the extra-cellular phase was monitored as an increase in absorbance at $340 \mathrm{~nm}$. As a control, the ATP synthase inhibitor oligomycin (OLIGO, $5 \mu \mathrm{g} / 10 \mu \mathrm{l}$ ) was added during the reaction to ascertain the inhibition of the mitochondrial ATP production. Attention was reserved to use excess HK/G6P$\mathrm{DH}$ coupled enzymes to guarantee a non-limiting ADP-restoring system for the assay of ATP production.

\section{Measurement of ATP Levels in ciPTEC}

ciPTEC were detached from plate, washed with PBS and cellular ATP was extracted by using the boiling water procedure, as described in Yang et al. (2002). The amount of intracellular ATP was determined enzymatically in the extracts, as described in Valenti et al. (2010). Cells were left under basal condition or stimulated with NPS-R568 (10 $\mu \mathrm{M}$ for $60 \mathrm{~min}$ at $\left.37^{\circ} \mathrm{C}\right)$ in Ringer's solution containing $120 \mathrm{mM} \mathrm{NaCl}, 4 \mathrm{mM} \mathrm{KCl}, 15 \mathrm{mM} \mathrm{NaHCO}_{3}$, $1 \mathrm{mM} \mathrm{MgCl}_{2}, 15 \mathrm{mM}$ Hepes, $0.5 \mathrm{mM} \mathrm{NaH}_{2} \mathrm{PO}_{4}, 10 \mathrm{mM}$ Glucose, $2 \mathrm{mM} \mathrm{CaCl}_{2}, \quad 0.5 \mathrm{mM} \mathrm{Na}_{2} \mathrm{HPO}_{4}, 0.4 \mathrm{mM} \mathrm{MgSO} 4, \mathrm{pH} 7.4$ (modified by Mekahli et al., 2012; Miyakawa et al., 2013).

\section{Fluorescence Resonance Energy Transfer (FRET) Measurements}

To evaluate mitochondrial calcium content, fluorescence resonance energy transfer (FRET) experiments were performed as described (Di Mise et al., 2015, 2018). Briefly, ciPTEC were seeded onto $20-\mathrm{mm}$ glass coverslips at $37^{\circ} \mathrm{C}$ for 11 days and were transiently transfected with a plasmid encoding a mitochondrially targeted cameleon containing a mutant calmodulin $(\mathrm{mCaM})$ sequence cloned between CFP and circularly permuted Venus (cpV) (Palmer et al., 2006). Experiments were performed $24 \mathrm{~h}$ post-transfection. Cells were left under basal condition or stimulated with NPS-R568 $\left(10 \mu \mathrm{M}\right.$ for $60 \mathrm{~min}$ at $37^{\circ} \mathrm{C}$ ) in Ringer's solution, described above, containing $2 \mathrm{mM} \mathrm{CaCl}_{2}$. 
FRET measurements were carried out using MetaMorph software (Molecular Devices, MDS Analytical Technologies, Toronto, Canada). CFP and YFP were excited at 436 and $500 \mathrm{~nm}$, respectively; fluorescence emitted was measured at $480 / 40 \mathrm{~nm}$ for CFP and 535/30 nm for YFP and FRET. Corrected normalized FRET values were determined as already described (Rodighiero et al., 2008; Tamma et al., 2013, 2017; Russo et al., 2017). Each image was corrected for CFP cross-talk and YFP cross-excitation. Therefore, netFRET $=$ [IFRETbg - ICFPbg.K1 - IYFPbg. $(\mathrm{K} 2-$ $\alpha \mathrm{K} 1)] /(1-\delta \mathrm{K} 1)$ where IFRETbg, ICFPbg, and IYFPbg are the background-corrected pixel gray values measured in the FRET, $\mathrm{CFP}$, and YFP windows, respectively; K1, K2, $\alpha$, and $\delta$ are calculated to evaluate the crosstalk between donor and acceptor. The integrated fluorescence density values of the images from each cell were analyzed using MetaMorph and Microsoft Excel software.

\section{Immunofluorescence Microscopy}

CaSR immunofluorescence localization in polarized ciPTEC was performed as previously described (Jansen et al., 2014; Di Mise et al., 2015). ciPTEC were cultured on polyester Transwell inserts, left 11 days at $37^{\circ} \mathrm{C}$ for maturation, and then fixed using $2 \%(\mathrm{w} / \mathrm{v})$ paraformaldehyde in HBSS with addition of $2 \%(\mathrm{w} / \mathrm{v})$ sucrose for $5 \mathrm{~min}$ and permeabilized in $0.3 \%(\mathrm{v} / \mathrm{v})$ triton X-100 in HBSS for $10 \mathrm{~min}$.

Cells were incubated with antibodies diluted in block solution containing $2 \%(\mathrm{w} / \mathrm{v})$ bovine serum albumin (BSA) and $0.1 \%(\mathrm{v} / \mathrm{v})$ tween-20 in HBSS against the calcium-sensing receptor (CaSR) at $4^{\circ} \mathrm{C}$ overnight. Following treatment with secondary rabbit-antimouse-biotin antibodies followed by Streptavidin-488, samples were mounted on glass slides with Mowiol. Images were obtained with a confocal microscope Leica TCS SP2 (Leica Microsystems, Heerbrugg, Switzerland).

\section{Statistical Analysis}

Statistical analysis was performed using One-way ANOVA followed by Newman-Keuls multiple comparisons test or Twoway ANOVA followed by Turkey's multiple comparisons test or $t$-test. All values are expressed as means \pm SEM. A difference of $P<0.05$ was considered statistically significant.

\section{RESULTS AND DISCUSSION}

\section{Impaired Bioenergetics Status in ciPTEC Silenced for PC1}

Emerging evidence of inherent metabolic reprogramming in PKD1 knockout cells suggests that the PC1-PC2 complex regulates cellular metabolism (Menezes et al., 2016; Padovano et al., 2017). The exact nature of the metabolic alterations remains controversial, with some groups reporting enhanced glycolysis, reminiscent of the Warburg phenomenon (Rowe et al., 2013; Padovano et al., 2017), and others reporting no evidence for a glycolytic switch (Menezes et al., 2016; Warner et al., 2016), proposing instead fatty acid oxidation impairment (Menezes et al., 2016; Hajarnis et al., 2017).

Lin and collaborators (Lin et al., 2018) suggest a direct link between PKD proteins and control of mitochondrial activity, showing that PKD1 knockout cells have different metabolic fluxes with likely altered oxidoreductase activity, consistent with changes in $\mathrm{NAD}^{+} / \mathrm{NADH}$ ratio. Furthermore, their data document altered mitochondrial membrane potential and abnormal mitochondrial networks in PKD1 knockout cell lines. Morphological abnormalities of mitochondria have also been found in human ADPKD cyst-derived cells with heterozygous and homozygous PKD1 mutation, indicated to facilitate cyst formation in ADPKD (Ishimoto et al., 2017).

On this basis, to investigate whether the bioenergetics status is affected in ciPTEC stably silenced for PC1, we first evaluated the mitochondrial ATP synthesis in ciPTEC-PC1KD cells compared with wild type clone, by monitoring in continuous the ATP produced by mitochondria in situ and flowed outside cells, under conditions where oxidative phosphorylation (OXPHOS) can take place (Valenti et al., 2010). ciPTEC-PC1KD or ciPTECwt, permeabilized with digitonin $(0.01 \%)$, were incubated with the ATP detecting system (ATP-ds) and the efflux of ATP in the extracellular phase occurring after the addition of ADP was monitored as an increase in absorbance at $340 \mathrm{~nm}$ due to NADPH synthesis, and evaluated as a measure of mitochondrial ATP production (Figure 1A).

To give a more complete evidence on the efficiency of the OXPHOS system, and disclose the distinctive contribution of each mitochondrial respiratory chain complex composing the OXPHOS apparatus, the mitochondrial ATP synthesis was measured by supplying as energy sources the respiratory substrates of complex I glutamate plus malate (GLU+MAL), complex II succinate (SUCC) or complex IV ascorbate plus TMPD (ASC+TMPD). As shown by the representative spectrophotometric traces (Figures 1B-D) and by the histograms reporting the statistical analyses of data (Figure 2A), a drastic reduction in the rate of mitochondrial ATP synthesis was found, commonly shared when supplied ciPTEC-PC1KD with GLU+MAL (estimated as $41 \pm 13 \%$ vs. ciPTECwt, $P<0.01$ ) or SUCC (estimated as $39 \pm 10 \%$ vs. ciPTECwt, $P<0.01$ ) or ASC+TMPD (estimated as $56 \pm 11 \%$ vs. ciPTECwt, $P<0.01$ ), thus indicating a multilevel inhibition of the mitochondrial ATP production by OXPHOS in ciPTEC-PC1KD.

To assess whether the compromised mitochondrial energy efficiency found in ciPTEC-PC1KD could impact the cell energy status, the cellular ATP pool was measured (Figure 2B). ATP level was found significantly reduced in ciPTEC-PC1KD (estimated as $38 \pm 4 \%, P<0.0001$ ) with respect to wt, suggesting that the deficit in ATP production by mitochondria reflects and mirror the overall cellular energy level in ciPTEC-PC1KD.

These data indicate an impairment in mitochondrial OXPHOS associated with a great shortage in cell energy status in PKD1 silenced cells. How the lack of PKD1 causes such a mitochondrial dysfunction in ciPTEC it is not yet clear. Both transcriptional and/or post-translational mechanisms regulating OXPHOS process may be involved. Among post-translational regulation of OXPHOS, calcium signaling between cytosol and matrix primarily coordinates the crosstalk of mitochondria within the cell, establishing the balance between the energy requests of the cell and the energy production by mitochondrial OXPHOS (Glancy and Balaban, 2012). 


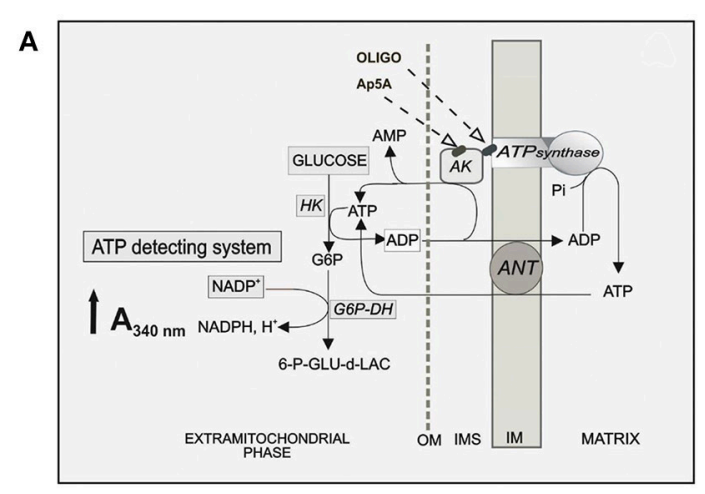

B



C



D

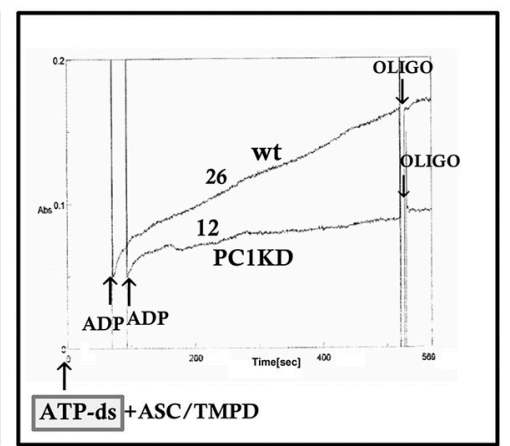

FIGURE 1 | Impaired mitochondrial ATP synthesis in human ciPTEC-PC1KD. (A) Schematic representation of the mitochondrial ATP synthesis revealed by ATP detecting system (ATP-ds). ANT, adenine nucleotide translocator; AK, adenylate kinase; HK, hexokinase; G6P-DH, glucose 6-phosphate dehydrogenase; 6P-GLUC$\delta$-LAC, 6 phosphogluconate- $\delta$-lactone; IMS, intermembrane space; MIM, mitochondrial inner membrane; OM, outer membrane. (B-D) Representative spectrophotometric traces of mitochondrial ATP production in human ciPTEC. Either human ciPTEC stably silenced for polycystin-1 (ciPTEC-PC1KD) or wild type clone (ciPTECwt) $(0.3 \mathrm{mg})$ were permeabilized with $0.01 \%$ digitonin for $5 \mathrm{~min}$ and incubated at $37^{\circ} \mathrm{C}$ in $2 \mathrm{ml}$ of respiration medium in the presence of ATP-ds plus the indicated respiratory substrates. (B) Glutamate plus malate (GLU/MAL, $5 \mathrm{mM}$ each), (C) $5 \mathrm{mM}$ succinate (SUCC) plus $3 \mu \mathrm{M}$ rotenone, (D) $5 \mathrm{mM}$ ascorbate plus $0.5 \mathrm{mM}$ TMPD (ASC/TMPD). Where indicated, ADP $(0.5 \mathrm{mM})$ was added. At the arrows, the ATP synthase inhibitor oligomycin (OLIGO, $5 \mu \mathrm{g} / 10 \mu \mathrm{l})$ was added in course of reaction. The numbers along curves represent the rates of the increase in absorbance at $340 \mathrm{~nm}$, measured as tangents to the initial slopes and expressed as nmol of $\mathrm{NADPH}$ formed/min per $\mathrm{mg}$ of protein.
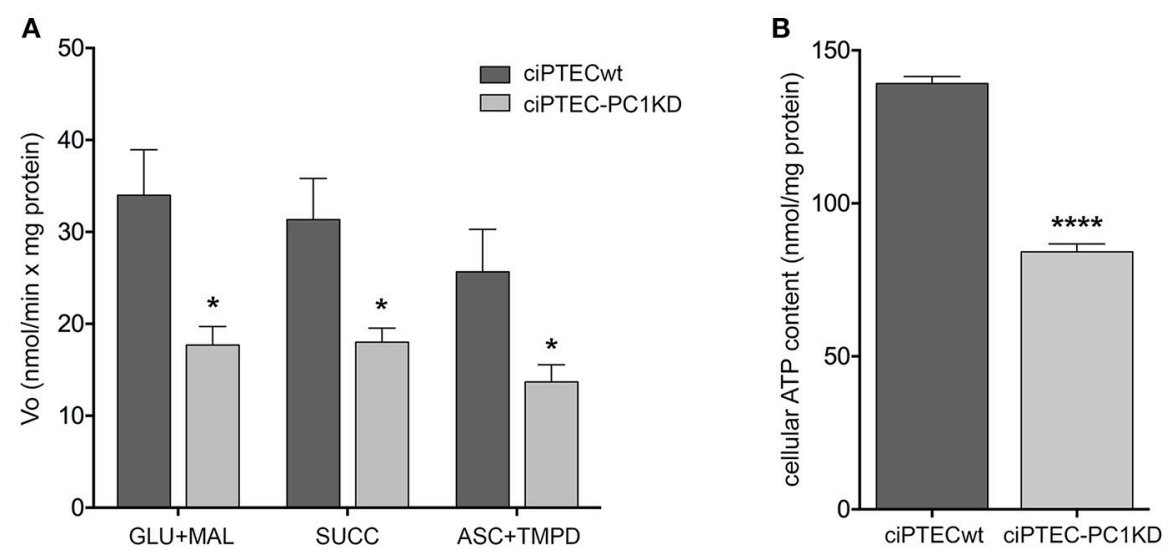

FIGURE 2 | Impaired bioenergetics status in human ciPTEC-PC1KD. (A) The rate of mitochondrial ATP production was measured in human ciPTEC stably silenced for polycystin-1 (CiPTEC-PC1KD) vs. wild type clone (ciPTECwt), in the presence of the respiratory substrates GLU plus MAL (GLU/MAL, $5 \mathrm{mM}$ each) or $5 \mathrm{mM}$ SUCC plus $3 \mu \mathrm{M}$ rotenone, or $5 \mathrm{mM}$ ASC plus $0.5 \mathrm{mM}$ TMPD (ASC/TMPD), as described in the legend of Figure 1. Values are means \pm SEM $\left({ }^{*} P<0.01 \mathrm{vs}\right.$. ciPTECwt) obtained from three independent experiments and expressed as nmol NADPH/min per mg protein. (B) Cellular ATP levels were measured in ciPTEC-PC1KD vs. matched ciPTECwt, as described under Methods. Each histogram is representative of four independent experiments. Data are expressed as means \pm SEM. Significant differences with respect to wt were calculated by Student's $t$-test $\left.{ }^{\star \star \star \star} P<0.0001\right)$. 


\section{Reduced Mitochondrial Calcium Content in CiPTEC-PC1KD}

It has been shown that cultured epithelial cells derived from human ADPKD cysts exhibit a reduction in steady state cytosolic calcium levels with respect to normal human kidney cells (Yamaguchi et al., 2006). Moreover, PC complex interacts with other calcium channels expressed in the ER, displaying a pivotal role in the prevention of intracellular stores depletion, especially of the ER itself (Anyatonwu et al., 2007; Weber et al., 2008; Santoso et al., 2011). Recently, we demonstrated that, compared to ciPTECwt, ciPTEC-PC1KD have significantly lower calcium concentration both in the cytosol and in the ER, highlighting that the loss-of-function mutation in PKD1 is strictly connected to the dysregulation of the two intracellular calcium bulks, likely secondary to the PC complex disruption and dysfunction (Di Mise et al., 2018).

The ER is known to be functionally associated to mitochondria with a rapid transport of calcium across their membranes and its accumulation in the mitochondrial matrix, where several calcium effectors are located (Tarasov et al., 2012). The inositol 1,4,5-trisphosphate receptors (IP3Rs) are the main drivers of the ER-to-mitochondria $\mathrm{Ca}^{2+}$ shuttle supporting cellular bioenergetics (Cárdenas et al., 2010). Mitochondrial calcium uptake by IP3R-released $\mathrm{Ca}^{2+}$ is fundamentally required to maintain adequate mitochondrial $\mathrm{NADH}$ production to sustain OXPHOS in resting cells (Cárdenas et al., 2010). IP3R activity is linked to regulation of cellular bioenergetics through calcium-dependent activation of mitochondrial ATP synthesis, by triggering key matrix enzymes, including pyruvate dehydrogenase, $\alpha$-oxoglutarate dehydrogenase, isocitrate dehydrogenase, as well as downstream elements of OXPHOS, comprising F1/Fo ATP synthase and the cytochrome chain (Glancy and Balaban, 2012).

In line with these findings, the resting mitochondrial calcium content in ciPTEC-PC1KD resulted significantly reduced with respect to wt cells (ciPTEC-PC1KD $=88.05 \pm 2.6 \%, n=140$, vs. ciPTECwt $=100 \%, n=192 ; P<0.01$; Figure 3). These data further confirm the functional interaction between ER and mitochondria that are in close apposition, allowing calcium transfer between these organelles and supporting the hypothesis that the PCs are involved in the regulation of mitochondrial calcium levels, as previously suggested (Patergnani et al., 2011; Padovano et al., 2017). Indeed, in line with this hypothesis, PCs have been shown to co-fractionate with mitochondria-associated ER membranes (MAMs) (Padovano et al., 2017).

\section{Activation of CaSR by NPS-R568 Results in Full Recovery of Mitochondrial Calcium Levels and Restoring of Cell Energy Deficit in ciPTEC-PC1KD}

ciPTEC stably silenced for PC1 as well as wild type clone, express endogenous CaSR mainly localized to the apical plasma membrane as it occurs in native renal proximal tubule epithelial cells (Figure 4) (Riccardi and Valenti, 2016; Di Mise et al., 2018). CaSR is coupled to three main groups of heterotrimeric G proteins, $G_{q / 11}, G_{i}$, and $G_{12 / 13}$. Recently we have shown that

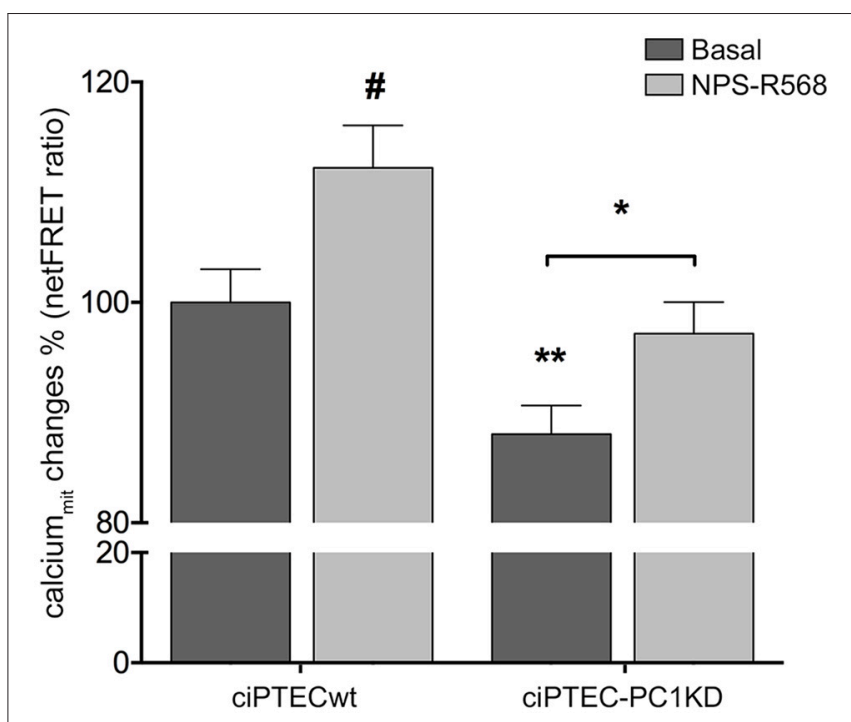

FIGURE 3 | Reduced mitochondrial calcium levels in human ciPTEC-PC1KD: effect of CaSR positive allosteric modulator NPS-R568. Evaluation of mitochondrial $\mathrm{Ca}^{2+}$ with mitochondria-targeted cameleon (4mtD3cpV) FRET probe. Histogram compares changes in normalized FRET (netFRET) ratio between ciPTECwt and ciPTEC-PC1KD, at basal conditions or treated with NPS-R568. At rest, ciPTEC-PC1KD presented a significant lower mitochondrial calcium content with respect to ciPTECwt $\left({ }^{* *} P<0.01\right)$. CaSR stimulation with NPS-R568 $10 \mu \mathrm{M}$ induced a significant increase in calcium levels in ciPTEC-PC1KD, restoring levels close to ciPTECwt at basal conditions. All data were analyzed by One-way ANOVA followed by Newman-Keuls multiple comparisons test and expressed as means \pm SEM $\left({ }^{*} P<0.01\right.$ ciPTEC-PC1KD+NPS-R568 vs. ciPTEC-PC1KD Basal; $\# P<0.001$ ciPTECwt+NPS-R568 vs. ciPTECwt Basal).

CaSR expressed in ciPTEC interacts with $\mathrm{G}_{\mathrm{q}}$ as a downstream effector (Di Mise et al., 2015). As known, when CaSR is activated, it induces cytosolic calcium increase via $\mathrm{G}_{\mathrm{q}}$ activation which stimulates PLC with subsequent IP3 and diacylglycerol production. IP3 binds to high affinity receptors on the ER (IP3Rs), causing the release of calcium into the cytoplasm.

On this basis, we evaluated the effect of CaSR stimulation with NPS-R568 on mitochondrial calcium levels. NPS-R568 treatment resulted in a significant increase in ciPTEC-PC1KD mitochondrial calcium (ciPTEC-PC1KD+NPS-R568 $=97.17$ $\pm 2.87 \%, n=200$ ), which raised close to the basal levels observed in ciPTECwt (Figure 3). This result suggests that the increase in mitochondrial calcium is a consequence of the CaSR activation induced signaling which leads to IP3 production and calcium influx from ER to the mitochondrion through IP3Rs (Cárdenas et al., 2010). Moreover, the increase of cytosolic $\mathrm{Ca}^{2+}$ elicited by CaSR activation also contributes to the increment of mitochondrial $\mathrm{Ca}^{2+}$ content via the mitochondrial calcium uniporter (MCU) (Marchi and Pinton, 2014).

As known, mitochondrial calcium homeostasis and uptake has a pivotal role in the regulation of mitochondrial ATP generation as well as cytosolic $\mathrm{NAD}^{+} / \mathrm{NADH}$ metabolism, thus sustaining the energy requirements of the cell (Raffaello et al., 2016; Arduino and Perocchi, 2018). 
Remarkably, ciPTEC treatment with the calcimimetic NPSR568 resulted in a full recovery of cellular ATP content in ciPTEC-PC1KD (ciPTEC-PC1KD+NPS-R568 $=144.3 \pm$ $1.67 \mathrm{nmol} / \mathrm{mg}$ protein), restoring the levels measured in ciPTECwt at basal conditions (ciPTECwt $=139.2 \pm 2.27$ $\mathrm{nmol} / \mathrm{mg}$ protein; Figure $5 \mathbf{A}$ ). To assess the strict involvement of calcium transfer from ER to mitochondria in sustaining cellular bioenergetics thus rescuing energy deficit in PC1 knockdown cells after CaSR activation, ciPTEC were treated with 2-aminoethoxydiphenyl borate (2-APB), a selective IP3R inhibitor (Szatkowski et al., 2010). Furthermore, to abolish the calcium amount permeating mitochondria directly from the cytosol via MCU, we blocked IP3Rs with 2-APB in presence of BAPTA-AM, a cell-permeant highly selective calcium chelator (Gerbino et al., 2009). 2-APB treatment of ciPTEC-PC1KD completely prevented the rescue of bioenergetics deficit induced by NPS-R568, showing values comparable to the intracellular ATP content measured under basal conditions (70.6 \pm 0.9 $\mathrm{nmol} / \mathrm{mg}$ protein; Figure 5B). A similar reduction in cellular ATP was reported in ciPTECwt treated with 2 -APB $(82.4 \pm 0.82$



\section{CiPTEC-PC1KD}
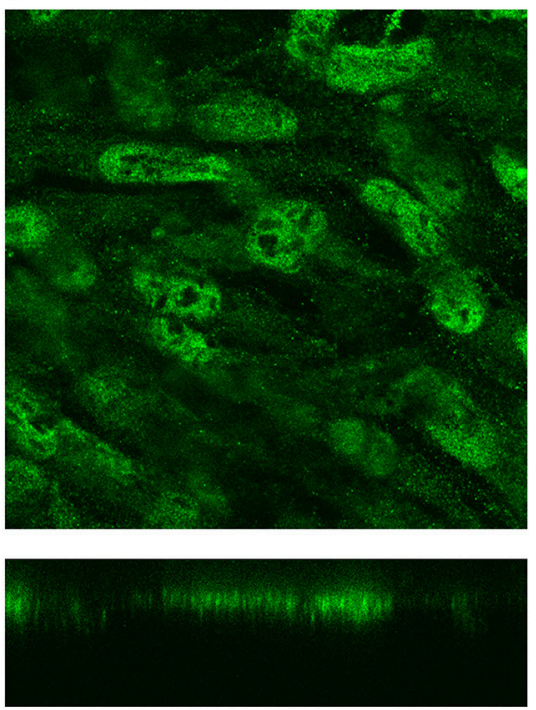

FIGURE 4 | Immunofluorescence localization of endogenous CaSR in human ciPTEC cells. In polarized ciPTEC, CaSR shows a predominant apical plasma membrane localization as occurs in native proximal tubule epithelial cells.


FIGURE 5 | NPS-R568 treatment results in a complete recovery of cell energy status in human ciPTEC-PC1KD. The ATP level in human ciPTEC was measured in three independent experiments for each experimental group and expressed as nmol ATP/mg protein. (A) CaSR stimulation elicited by NPS-R568 induced a significant increase in cellular ATP content in ciPTEC-PC1KD, restoring levels comparable to ciPTECwt at basal conditions. Data were analyzed by One-way ANOVA followed by Newman-Keuls multiple comparisons test and are expressed as means \pm SEM $\left(^{\star \star \star \star} P<0.0001\right.$ vs. ciPTECwt Basal or ciPTEC-PKD1+NPS-R568). (B) IP3R inhibition with 2-APB in ciPTEC-PC1KD prevented cellular ATP levels rescue induced by CaSR activation. Data were analyzed by Two-way ANOVA followed by Turkey's multiple comparisons test and are expressed as means \pm SEM ( ${ }^{\star \star \star *} P<0.0001$ vs. NPS-R568 treatment in each cell line). 




nmol/mg protein; Figure 5B), suggesting a critical role of IP3Rs in driving ER-to-mitochondria $\mathrm{Ca}^{2+}$ shuttle supporting cellular bioenergetics. BAPTA-AM per se did not affect ATP cellular levels (data not shown).

These findings point to a crucial role of mitochondrial calcium in regulating the mitochondrial energy status. Indeed, calcium is actively transported inside mitochondria and accumulates in the mitochondrial matrix, where several calcium protein targets are located (Rizzuto et al., 2012) (details in the model in Figure 6). In this context, the existence of high-calcium micro-domains between ER and mitochondria ensures efficient calcium transfer from ER to mitochondria 
(Rizzuto et al., 1998; Csordás et al., 1999, 2010), which is mediated by a multiprotein complex composed-among others-of the IP3R at ER membrane and of the VDAC at outer mitochondrial membrane (OMM) (Szabadkai et al., 2006). The zones of close contact between the ER and mitochondria, called Mitochondria Associated Membranes (MAMs), are crucial for a correct crosstalk between the two organelles (Csordás et al., 2006). Therefore, MAMs disruption causes the suppression of the IP3-mediated release of calcium from the ER to mitochondria, with a consequent reduction of ATP production (Rowland and Voeltz, 2012).

A model of intracellular calcium signaling network in PC1 deficient cells exposed to calcimimetic is shown in Figure 6.

To summarize, the present contribution provides the first evidence that selective CaSR activation in human ciPTEC stably knocked down for PC1, restores mitochondrial calcium content and fully rescues the bioenergetics dysfunction known to facilitate cysts formation in ADPKD. These data, together with our previous demonstration that CaSR activation in ciPTECPC1KD increases cytosolic calcium and decreases cAMP and mTOR activity, indicate that CaSR signaling can reverse the principal dysregulations considered the most proximal events

\section{REFERENCES}

Anyatonwu, G. I., Estrada, M., Tian, X., Somlo, S., and Ehrlich, B. E. (2007). Regulation of ryanodine receptor-dependent calcium signaling by polycystin-2. Proc. Natl. Acad. Sci. U.S.A. 104, 6454-6459. doi: 10.1073/pnas.0610324104

Arduino, D. M., and Perocchi, F. (2018). Pharmacological modulation of mitochondrial calcium homeostasis. J. Physiol. 596, 2717-2733. doi: 10.1113/JP274959

Babich, V., Zeng, W. Z., Yeh, B. I., Ibraghimov-Beskrovnaya, O., Cai, Y., Somlo, S., et al. (2004). The N-terminal extracellular domain is required for polycystin-1-dependent channel activity. J. Biol. Chem. 279, 25582-25589. doi: 10.1074/jbc.M402829200

Cárdenas, C., Miller, R. A., Smith, I., Bui, T., Molgó, J., Müller, M., et al. (2010). Essential regulation of cell bioenergetics by constitutive $\mathrm{InsP} 3$ receptor $\mathrm{Ca} 2+$ transfer to mitochondria. Cell 142, 270-283. doi: 10.1016/j.cell.2010.06.007

Celić, A., Petri, E. T., Demeler, B., Ehrlich, B. E., and Boggon, T. J. (2008). Domain mapping of the polycystin-2 C-terminal tail using de novo molecular modeling and biophysical analysis. J. Biol. Chem. 283, 28305-28312. doi: 10.1074/jbc.M802743200

Celić, A. S., Petri, E. T., Benbow, J., Hodsdon, M. E., Ehrlich, B. E., and Boggon, T. J. (2012). Calcium-induced conformational changes in C-terminal tail of polycystin-2 are necessary for channel gating. J. Biol. Chem. 287, 17232-17240. doi: 10.1074/jbc.M112.354613

Chapin, H. C., and Caplan, M. J. (2010). The cell biology of polycystic kidney disease. J. Cell Biol. 191, 701-710. doi: 10.1083/jcb.201006173

Chapin, H. C., Rajendran, V., and Caplan, M. J. (2010). Polycystin-1 surface localization is stimulated by polycystin-2 and cleavage at the G protein-coupled receptor proteolytic site. Mol. Biol. Cell 21, 4338-4348. doi: $10.1091 / \mathrm{mbc}$.e10-05-0407

Chauvet, V., Tian, X., Husson, H., Grimm, D. H., Wang, T., Hiesberger, T., et al. (2004). Mechanical stimuli induce cleavage and nuclear translocation of the polycystin-1 C terminus. J. Clin. Invest. 114, 1433-1443. doi: 10.1172/JCI21753

Csordás, G., Renken, C., Várnai, P., Walter, L., Weaver, D., Buttle, K. F., et al. (2006). Structural and functional features and significance of the physical linkage between ER and mitochondria. J. Cell Biol. 174, 915-921. doi: $10.1083 /$ jcb. 200604016 in ADPKD pathogenesis, making CaSR a potential candidate as therapeutic target.

\section{AUTHOR CONTRIBUTIONS}

GV and DV designed research and supervised the project. DV and $\mathrm{AD}$ designed, performed, and interpreted experiments. MR, $\mathrm{MC}, \mathrm{MV}$, and GT performed research and analyzed data. AD, GV, and DV wrote the paper. All authors commented on the manuscript.

\section{FUNDING}

This study was supported in part by Telethon funding (grant number GGP13227) and by ASI (Italian Space Agency, grant number 2013-091-R.0).

\section{ACKNOWLEDGMENTS}

We thank Dr. Elena Levtchenko for providing ciPTECwt and ciPTEC-PC1KD cell lines. The authors thank Amgen (Amgen Dompé S.p.a., Milan, Italy) for providing NPS-R568.

Csordás, G., Thomas, A. P., and Hajnóczky, G. (1999). Quasi-synaptic calcium signal transmission between endoplasmic reticulum and mitochondria. EMBO J. 18, 96-108. doi: 10.1093/emboj/18.1.96

Csordás, G., Várnai, P., Golenár, T., Roy, S., Purkins, G., Schneider, T. G., et al. (2010). Imaging interorganelle contacts and local calcium dynamics at the ER-mitochondrial interface. Mol. Cell 39, 121-132. doi: 10.1016/j.molcel.2010.06.029

Delmas, P., Nauli, S. M., Li, X., Coste, B., Osorio, N., Crest, M., et al. (2004). Gating of the polycystin ion channel signaling complex in neurons and kidney cells. FASEB J. 18, 740-742. doi: 10.1096/fj.03-0319fje

Di Mise, A., Tamma, G., Ranieri, M., Centrone, M., Van Den Heuvel, L., Mekahli, D., et al. (2018). Activation of calcium-sensing receptor increases intracellular calcium and decreases cAMP and mTOR in PKD1 deficient cells. Sci. Rep. 8:5704. doi: 10.1038/s41598-018-23732-5

Di Mise, A., Tamma, G., Ranieri, M., Svelto, M., Heuvel, B., Levtchenko, E. N., et al. (2015). Conditionally immortalized human proximal tubular epithelial cells isolated from the urine of a healthy subject express functional calcium-sensing receptor. Am. J. Physiol. Renal Physiol. 308, F1200-F1206. doi: 10.1152/ajprenal.00352.2014

Feng, S., Okenka, G. M., Bai, C. X., Streets, A. J., Newby, L. J., Dechant, B. T., et al. (2008). Identification and functional characterization of an N-terminal oligomerization domain for polycystin-2. J. Biol. Chem. 283, 28471-28479. doi: 10.1074/jbc.M803834200

Geng, L., Okuhara, D., Yu, Z., Tian, X., Cai, Y., Shibazaki, S., et al. (2006). Polycystin-2 traffics to cilia independently of polycystin-1 by using an Nterminal RVxP motif. J. Cell Sci. 119, 1383-1395. doi: 10.1242/jcs.02818

Gerbino, A., Ranieri, M., Lupo, S., Caroppo, R., Debellis, L., Maiellaro, I., et al. (2009). Ca2+-dependent $\mathrm{K}+$ efflux regulates deoxycholate-induced apoptosis of BHK-21 and Caco-2 cells. Gastroenterology 137, 955-964, e1-2. doi: 10.1053/j.gastro.2009.03.038

Glancy, B., and Balaban, R. S. (2012). Role of mitochondrial Ca2+ in the regulation of cellular energetics. Biochemistry 51, 2959-2973. doi: 10.1021/bi2018909

Hajarnis, S., Lakhia, R., Yheskel, M., Williams, D., Sorourian, M., Liu, X., et al. (2017). MicroRNA-17 family promotes polycystic kidney disease progression through modulation of mitochondrial metabolism. Nat. Commun. 8:14395. doi: $10.1038 /$ ncomms 14395 
Hanaoka, K., Qian, F., Boletta, A., Bhunia, A. K., Piontek, K., Tsiokas, L., et al. (2000). Co-assembly of polycystin-1 and-2 produces unique cation-permeable currents. Nature 408, 990-994. doi: 10.1038/35050128

Harris, P. C., and Torres, V. E. (2014). Genetic mechanisms and signaling pathways in autosomal dominant polycystic kidney disease. J. Clin. Invest. 124, 2315-2324. doi: 10.1172/JCI72272

Harris, P. C., Ward, C. J., Peral, B., and Hughes, J. (1995). Polycystic kidney disease. 1: identification and analysis of the primary defect. J. Am. Soc. Nephrol. 6, 1125-1133.

Hughes, J., Ward, C. J., Peral, B., Aspinwall, R., Clark, K., San Millan, J. L., et al. (1995). The polycystic kidney disease 1 (PKD1) gene encodes a novel protein with multiple cell recognition domains. Nat. Genet. 10, 151-160. doi: 10.1038/ng0695-151

Ishimoto, Y., Inagi, R., Yoshihara, D., Kugita, M., Nagao, S., Shimizu, A., et al. (2017). Mitochondrial abnormality facilitates cyst formation in autosomal dominant polycystic kidney disease. Mol. Cell. Biol. doi: 10.1128/MCB.00337-17. [Epub ahead of print].

Jansen, J., Schophuizen, C. M., Wilmer, M. J., Lahham, S. H., Mutsaers, H. A., Wetzels, J. F., et al. (2014). A morphological and functional comparison of proximal tubule cell lines established from human urine and kidney tissue. Exp. Cell Res. 323, 87-99. doi: 10.1016/j.yexcr.2014.02.011

Klawitter, J., Reed-Gitomer, B. Y., Mcfann, K., Pennington, A., Klawitter, J., Abebe, K. Z., et al. (2014). Endothelial dysfunction and oxidative stress in polycystic kidney disease. Am. J. Physiol. Renal Physiol. 307, F1198-F1206. doi: 10.1152/ajprenal.00327.2014

Koulen, P., Cai, Y., Geng, L., Maeda, Y., Nishimura, S., Witzgall, R., et al. (2002). Polycystin-2 is an intracellular calcium release channel. Nat. Cell Biol. 4, 191-197. doi: 10.1038/ncb754

Li, Q. W., Lu, X. Y., You, Y., Sun, H., Liu, X. Y., Ai, J. Z., et al. (2012). Comparative proteomic analysis suggests that mitochondria are involved in autosomal recessive polycystic kidney disease. Proteomics 12, 2556-2570. doi: 10.1002/pmic.201100590

Lin, C. C., Kurashige, M., Liu, Y., Terabayashi, T., Ishimoto, Y., Wang, T., et al. (2018). A cleavage product of Polycystin-1 is a mitochondrial matrix protein that affects mitochondria morphology and function when heterologously expressed. Sci. Rep. 8:2743. doi: 10.1038/s41598-018-20856-6

Löhning, C., Pohlschmidt, M., Glücksmann-Kuis, M. A., Duyk, G., Bork, P., Schneider, M. O., et al. (1996). Structural motifs of the PKD1 protein. Nephrol. Dial. Transplant. 11(Suppl. 6), 2-4. doi: 10.1093/ndt/11.supp6.2

Magistroni, R., and Boletta, A. (2017). Defective glycolysis and the use of 2-deoxyD-glucose in polycystic kidney disease: from animal models to humans. J. Nephrol. 30, 511-519. doi: 10.1007/s40620-017-0395-9

Marchi, S., and Pinton, P. (2014). The mitochondrial calcium uniporter complex: molecular components, structure and physiopathological implications. J. Physiol. 592, 829-839. doi: 10.1113/jphysiol.2013.268235

Mekahli, D., Sammels, E., Luyten, T., Welkenhuyzen, K., Van Den Heuvel, L. P., Levtchenko, E. N., et al. (2012). Polycystin-1 and polycystin-2 are both required to amplify inositol-trisphosphate-induced $\mathrm{Ca} 2+$ release. Cell Calcium 51, 452-458. doi: 10.1016/j.ceca.2012.03.002

Menezes, L. F., Lin, C. C., Zhou, F., and Germino, G. G. (2016). Fatty acid oxidation is impaired in an orthologous mouse model of autosomal dominant polycystic kidney disease. EBioMedicine 5, 183-192. doi: 10.1016/j.ebiom.2016.01.027

Menon, V., Rudym, D., Chandra, P., Miskulin, D., Perrone, R., and Sarnak, M. (2011). Inflammation, oxidative stress, and insulin resistance in polycystic kidney disease. Clin. J. Am. Soc. Nephrol. 6, 7-13. doi: 10.2215/CJN.04140510

Miyakawa, A., Ibarra, C., Malmersjö, S., Aperia, A., Wiklund, P., and Uhlen, P. (2013). Intracellular calcium release modulates polycystin-2 trafficking. BMC Nephrol. 14:34. doi: 10.1186/1471-2369-14-34

Mochizuki, T., Wu, G., Hayashi, T., Xenophontos, S. L., Veldhuisen, B., Saris, J. J., et al. (1996). PKD2, a gene for polycystic kidney disease that encodes an integral membrane protein. Science 272, 1339-1342. doi: 10.1126/science.272.5266.1339

Oatley, P., Stewart, A. P., Sandford, R., and Edwardson, J. M. (2012). Atomic force microscopy imaging reveals the domain structure of polycystin-1. Biochemistry 51, 2879-2888. doi: 10.1021/bi300134b

O'hare, M. J., Bond, J., Clarke, C., Takeuchi, Y., Atherton, A. J., Berry, C., et al. (2001). Conditional immortalization of freshly isolated human mammary fibroblasts and endothelial cells. Proc. Natl. Acad. Sci. U.S.A. 98, 646-651. doi: 10.1073/pnas.98.2.646
Ong, A. C., and Wheatley, D. N. (2003). Polycystic kidney disease-the ciliary connection. Lancet 361, 774-776. doi: 10.1016/S0140-6736(03)12662-1

Padovano, V., Kuo, I. Y., Stavola, L. K., Aerni, H. R., Flaherty, B. J., Chapin, H. C., et al. (2017). The polycystins are modulated by cellular oxygen-sensing pathways and regulate mitochondrial function. Mol. Biol. Cell 28, 261-269. doi: 10.1091/mbc.e16-08-0597

Palmer, A. E., Giacomello, M., Kortemme, T., Hires, S. A., Lev-Ram, V., Baker, D., et al. (2006). Ca2+ indicators based on computationally redesigned calmodulin-peptide pairs. Chem. Biol. 13, 521-530. doi: 10.1016/j.chembiol.2006.03.007

Patergnani, S., Suski, J. M., Agnoletto, C., Bononi, A., Bonora, M., De Marchi, E., et al. (2011). Calcium signaling around Mitochondria Associated Membranes (MAMs). Cell Commun. Signal. 9:19. doi: 10.1186/1478-811X-9-19

Pazour, G. J., and Rosenbaum, J. L. (2002). Intraflagellar transport and cilia-dependent diseases. Trends Cell Biol. 12, 551-555. doi: 10.1016/S0962-8924(02)02410-8

Qian, F., Germino, F. J., Cai, Y., Zhang, X., Somlo, S., and Germino, G. G. (1997). PKD1 interacts with PKD2 through a probable coiled-coil domain. Nat. Genet. 16, 179-183. doi: 10.1038/ng0697-179

Raffaello, A., Mammucari, C., Gherardi, G., and Rizzuto, R. (2016). Calcium at the center of cell signaling: interplay between endoplasmic reticulum, mitochondria, and lysosomes. Trends Biochem. Sci. 41, 1035-1049. doi: 10.1016/j.tibs.2016.09.001

Riccardi, D., and Valenti, G. (2016). Localization and function of the renal calciumsensing receptor. Nat. Rev. Nephrol. 12, 414-425. doi: 10.1038/nrneph.2016.59

Rieusset, J. (2018). The role of endoplasmic reticulum-mitochondria contact sites in the control of glucose homeostasis: an update. Cell Death Dis. 9:388. doi: 10.1038/s41419-018-0416-1

Rizzuto, R., De Stefani, D., Raffaello, A., and Mammucari, C. (2012). Mitochondria as sensors and regulators of calcium signalling. Nat. Rev. Mol. Cell Biol. 13, 566-578. doi: 10.1038/nrm3412

Rizzuto, R., Pinton, P., Carrington, W., Fay, F. S., Fogarty, K. E., Lifshitz, L. M., et al. (1998). Close contacts with the endoplasmic reticulum as determinants of mitochondrial Ca2+ responses. Science 280, 1763-1766. doi: $10.1126 /$ science.280.5370.1763

Rodighiero, S., Bazzini, C., Ritter, M., Fürst, J., Botta, G., Meyer, G., et al. (2008). Fixation, mounting and sealing with nail polish of cell specimens lead to incorrect FRET measurements using acceptor photobleaching. Cell. Physiol. Biochem. 21, 489-498. doi: 10.1159/000129642

Rowe, I., Chiaravalli, M., Mannella, V., Ulisse, V., Quilici, G., Pema, M., et al. (2013). Defective glucose metabolism in polycystic kidney disease identifies a new therapeutic strategy. Nat. Med. 19, 488-493. doi: 10.1038/nm.3092

Rowland, A. A., and Voeltz, G. K. (2012). Endoplasmic reticulum-mitochondria contacts: function of the junction. Nat. Rev. Mol. Cell Biol. 13, 607-625. doi: $10.1038 / \mathrm{nrm} 3440$

Russo, A., Ranieri, M., Di Mise, A., Dossena, S., Pellegrino, T., Furia, E., et al. (2017). Interleukin-13 increases pendrin abundance to the cell surface in bronchial NCI-H292 cells via Rho/actin signaling. Pflugers Arch. 469, 1163-1176. doi: 10.1007/s00424-017-1970-6

Santoso, N. G., Cebotaru, L., and Guggino, W. B. (2011). Polycystin-1, 2, and STIM1 interact with IP(3)R to modulate ER Ca release through the PI3K/Akt pathway. Cell. Physiol. Biochem. 27, 715-726. doi: 10.1159/000330080

Satchell, S. C., Tasman, C. H., Singh, A., Ni, L., Geelen, J., Von Ruhland, C. J., et al. (2006). Conditionally immortalized human glomerular endothelial cells expressing fenestrations in response to VEGF. Kidney Int. 69, 1633-1640. doi: 10.1038/sj.ki.5000277

Szabadkai, G., Bianchi, K., Várnai, P., De Stefani, D., Wieckowski, M. R., Cavagna, D., et al. (2006). Chaperone-mediated coupling of endoplasmic reticulum and mitochondrial Ca2+ channels. J. Cell Biol. 175, 901-911. doi: $10.1083 /$ jcb.200608073

Szatkowski, C., Parys, J. B., Ouadid-Ahidouch, H., and Matifat, F. (2010). Inositol 1,4,5-trisphosphate-induced $\mathrm{Ca} 2+$ signalling is involved in estradiol-induced breast cancer epithelial cell growth. Mol. Cancer 9:156. doi: 10.1186/1476-4598-9-156

Tamma, G., Di Mise, A., Ranieri, M., Geller, A., Tamma, R., Zallone, A., et al. (2017). The V2 receptor antagonist tolvaptan raises cytosolic calcium and prevents AQP2 trafficking and function: an in vitro and in vivo assessment. J. Cell. Mol. Med. 21, 1767-1780. doi: 10.1111/jcmm.13098 
Tamma, G., Ranieri, M., Dossena, S., Di Mise, A., Nofziger, C., Svelto, M., et al. (2013). A FRET-based approach for quantitative evaluation of forskolininduced pendrin trafficking at the plasma membrane in bronchial NCI H292 cells. Cell. Physiol. Biochem. 32, 200-209. doi: 10.1159/000356639

Tarasov, A. I., Griffiths, E. J., and Rutter, G. A. (2012). Regulation of ATP production by mitochondrial $\mathrm{Ca}(2+)$. Cell Calcium 52, 28-35. doi: $10.1016 /$ j.ceca.2012.03.003

Torres, V. E., Harris, P. C., and Pirson, Y. (2007). Autosomal dominant polycystic kidney disease. Lancet 369, 1287-1301. doi: 10.1016/S0140-6736(07)60601-1

Valenti, D., De Bari, L., De Rasmo, D., Signorile, A., Henrion-Caude, A., Contestabile, A., et al. (2016). The polyphenols resveratrol and epigallocatechin-3-gallate restore the severe impairment of mitochondria in hippocampal progenitor cells from a Down syndrome mouse model. Biochim. Biophys. Acta 1862, 1093-1104. doi: 10.1016/j.bbadis.2016.03.003

Valenti, D., Tullo, A., Caratozzolo, M. F., Merafina, R. S., Scartezzini, P., Marra, E., et al. (2010). Impairment of F1F0-ATPase, adenine nucleotide translocator and adenylate kinase causes mitochondrial energy deficit in human skin fibroblasts with chromosome 21 trisomy. Biochem. J. 431, 299-310. doi: 10.1042/BJ20100581

Warner, G., Hein, K. Z., Nin, V., Edwards, M., Chini, C. C., Hopp, K., et al. (2016). Food restriction ameliorates the development of polycystic kidney disease. J. Am. Soc. Nephrol. 27, 1437-1447. doi: 10.1681/ASN.2015020132

Weber, K. H., Lee, E. K., Basavanna, U., Lindley, S., Ziegelstein, R. C., Germino, G. G., et al. (2008). Heterologous expression of polycystin-1 inhibits endoplasmic reticulum calcium leak in stably transfected MDCK cells. Am. J. Physiol. Renal Physiol. 294, F1279-F1286. doi: 10.1152/ajprenal.00348. 2007

Wilmer, M. J., Saleem, M. A., Masereeuw, R., Ni, L., Van Der Velden, T. J., Russel, F. G., et al. (2010). Novel conditionally immortalized human proximal tubule cell line expressing functional influx and efflux transporters. Cell Tissue Res. 339, 449-457. doi: 10.1007/s00441-009-0882-y

Xu, C., Rossetti, S., Jiang, L., Harris, P. C., Brown-Glaberman, U., Wandinger-Ness, A., et al. (2007). Human ADPKD primary cyst epithelial cells with a novel, single codon deletion in the PKD1 gene exhibit defective ciliary polycystin localization and loss of flow-induced Ca2+ signaling. Am. J. Physiol. Renal Physiol. 292, F930-F945. doi: 10.1152/ajprenal.00285.2006

Yamaguchi, T., Hempson, S. J., Reif, G. A., Hedge, A. M., and Wallace, D. P. (2006). Calcium restores a normal proliferation phenotype in human polycystic kidney disease epithelial cells. J. Am. Soc. Nephrol. 17, 178-187. doi: 10.1681/ASN.2005060645

Yang, N. C., Ho, W. M., Chen, Y. H., and Hu, M. L. (2002). A convenient one-step extraction of cellular ATP using boiling water for the luciferin-luciferase assay of ATP. Anal. Biochem. 306, 323-327. doi: 10.1006/abio.2002.5698

Yu, Y., Ulbrich, M. H., Li, M. H., Buraei, Z., Chen, X. Z., Ong, A. C., et al. (2009). Structural and molecular basis of the assembly of the TRPP2/PKD1 complex. Proc. Natl. Acad. Sci. U.S.A. 106, 11558-11563. doi: 10.1073/pnas.0903684106

Conflict of Interest Statement: The authors declare that the research was conducted in the absence of any commercial or financial relationships that could be construed as a potential conflict of interest.

Copyright (C) 2018 Di Mise, Ranieri, Centrone, Venneri, Tamma, Valenti and Valenti. This is an open-access article distributed under the terms of the Creative Commons Attribution License (CC BY). The use, distribution or reproduction in other forums is permitted, provided the original author(s) and the copyright owner(s) are credited and that the original publication in this journal is cited, in accordance with accepted academic practice. No use, distribution or reproduction is permitted which does not comply with these terms. 